\title{
Patterns of Sexual Mixing and STIs in Intimate Partner Relationships among Female Sex Workers in Nepal
}

SK Singh, Alankar Malviya, Vipul Vaibhav Pandey*, Santosh Kumar Sharma and Nidhi Sharma

International Institute for Population Sciences, Deonar, Mumbai, India

\begin{abstract}
Background and objective: Varying degrees of sexual mixing in an intimate partner relationship which provides emotional support and social security, in addition to the economic benefits to female sex workers (FSWs) - often results in risky sexual behaviour (for example, unprotected sex), hence increasing the vulnerability of FSWs to sexually transmitted infections (STIs). This paper analyses the pattern of sexual mixing and the major covariates of STIs in intimate partner sexual relationships among FSWs in Nepal.
\end{abstract}

Method: The findings are based on information collected from $2901 \mathrm{FSWs}$, selected by using the modified timelocation cluster sampling as part of mapping and size estimation of MARPs across different districts of Nepal in year 2010-11.

Results: Findings reveal that almost 40 percent or two-fifths of FSWs in Nepal have commercial as well as noncommercial sexual partners. Further, 5.2 percent of the FSWs reported having more than one non-commercial partner along with commercial partners. Half of the FSWs in intimate partner relationships reported their last sexual encounter as unprotected, which was further lower among those currently married. Adjusted effects of socio-demographic and behavioural correlates portray that being married $(p<0.01)$ and having a higher client load-a proxy of poverty and powerlessness $(p<0.05)$-are significant determinants of unprotected sex, which may also be attributed to a lack of risk perception in intimate relationships. A higher incidence of unprotected sex further leads to substantially higher prevalence of STIs (35\%), which is disproportionately higher among those aged 30 and above, and among those having no formal education.

Conclusion: Findings of this study highlight the requirement of strategies to address sexual rights of FSWs within intimate partner relationships, in addition to the need for occupational empowerment of FSWs in order to combat prevalence of STIs and improving their quality of life.

Keywords: Sexual mixing; Intimate partner relationship; Female sex worker; Sexual risk; Coital frequency; Condom use; Sexually transmitted infections

\section{Background}

An estimated 75 million men in Asia visit sex workers and 10 million women cater to them. Add to these figures male Intravenous Drug Users (IDUs) and Men who have Sex with Men (MSM) - approximately 20 million-and we have a very large number of people who are at high risk of getting infected and, in turn, can potentially infect their partners [1]. Since most men who buy sex are either married or will get married, significant numbers of 'low risk' women who only have sex with their husbands are exposed to HIV. A conservative estimate by the Commission on AIDS in Asia put this number at 50 million women [2]. Evidence from several Asian countries shows that these women are either married or in long-term relationships with men who engage in high-risk sexual behaviours [3]. Studies in Asia show that almost all new infections among women are contracted through marriage [2]. In India alone, approximately 90 percent of the 1.7 million women living with HIV were infected by their husbands or partners of long- term relationships [4].

A report by a joint UN initiative (UNAIDS, UNDP, UNIFEM, UNFPA) with regional partners such as the ASEAN Foundation and networks of people living with HIV confirms this trend and points to rampant intimate partner violence across social and economic strata as an additional characteristic in the increase of intimate partner transmission of HIV [1]. Intimate partner violence (physical and or sexual) is a particular cause for concern as a very significant factor that compounds-both directly and indirectly-intimate partner transmission of HIV. In India, Bangladesh and Nepal, women who were exposed to intimate partner violence from husbands infected with HIV through unprotected sex with multiple partners were seven times more likely to acquire HIV compared to women not exposed to violence and whose husbands did not have sex with multiple partners [5]. Women's subjugation and their low status in society and within the house-hold give them very little power to negotiate safe sex. This makes it particularly difficult for women generally and 'married' women specifically to protect themselves from HIV exposure and ultimately infection through sex with their spouse/partner. The culture of silence that surrounds sex [6], gender inequality, violence, and discrimination that is so pervasive in the region exacerbates the problem [7].

Migration and mobility further facilitate the spread of HIV. Most migrants are separated from spouses and families, and are often pushed into poor working and living conditions without recreational activities, leading to isolation and stress. As a result, they have a high risk of

*Corresponding author: Vipul Vaibhav Pandey, Scholar, International Institute for Population Sciences, Deonar, Mumbai, Tel: +919820378356, E-mail: vipulvaibhav.pandey@gmail.com

Received October 06, 2015; Accepted December 16, 2015; Published December 22, 2015

Citation: Singh SK, Malviya A, Pandey VV, Sharma SK, Sharma N (2015) Patterns of Sexual Mixing and STIs in Intimate Partner Relationships among Female Sex Workers in Nepal. J AIDS Clin Res 6: 531. doi:10.4172/2155-6113.1000531

Copyright: (C) 2015 Singh SK, et al. This is an open-access article distributed under the terms of the Creative Commons Attribution License, which permits unrestricted use, distribution, and reproduction in any medium, provided the original author and source are credited. 
engaging in paid or casual sex, acquiring HIV, and passing it on to their partners when they return home infected [1].

There are three fundamental gender-related structural factors that interact to fuel intimate partner transmission. The first is gender inequality in access to productive resources, an inequality that favours men and creates an unequal balance of power in heterosexual relationships. This inequality makes it less likely that women will have control over sexual interactions and makes them more prone to be victims of domestic violence. It also makes them less likely to be able to negotiate condom use or fidelity in their relationships, or leave relationships that they perceive to be risky. The second factor that fuels intimate partner transmission is the prevalence of sexual norms that promote a hetero-normative view of male and female sexuality and the stigmatization and criminalization of sex work. This stigmatizes homosexuality and bisexuality, and upholds a double standard of appropriate sexual behaviour. The stigma associated with homosexuality and bisexuality forces men who have sex with men to keep their sexual behaviour and sexual preferences hidden, and submit to the social pressure to prove their heterosexuality by marrying unsuspecting women. For female sex workers, a subset of these women, the conditions are no different. It is the criminalization and stigmatization of sex work that puts them at greater risk of physical and sexual violence, and forces them to hide their identities or disperse into settings where it is difficult to reach them or provide health services. The stigma and criminalization also forces their male partners to hide their own identities, making it difficult to reach them for HIVprevention education. In all of these ways, prevalent sexual norms conspire to create the grounds for Intimate Partner Transmission. The third structural factor that increases women's vulnerability in longterm relationships in Asia is the culture of silence that shrouds sex and sexuality, despite more than two decades of exposure to the HIV virus. This culture creates the dichotomy of casual sex as compared to intimate partner sex. Casual sex is socially structured as furtive, free of social boundaries and thus, satisfying, while intimate partner sex is structured as obligatory, stable, for purposes of procreation and thus, not exciting. Due to this culture, women remain uninformed about sex and HIV prevention, and inter-partner communication on ways to make sex more satisfying and pleasurable is restricted [6].

An insight into the linkages between intimate partner relationship and STI/HIV vulnerability in a sex worker's life suggests that intimate relationships provide a space for feelings of inclusion and social safety. An intimate partner for sex workers acts as a support system. In conservative societies like Nepal, there is tremendous social stigma, occupational health hazards and societal exclusion that FSWs face due to their profession. On the other hand, the husbands or sexual partners who many times are the pimps or middle men act as shields for these women, protecting them from societal harassment, sometimes providing them the emotional support or family in terms of a spousal role. In such cases, involvement with clients irrespective of regular or occasional and on the other hand with husband or intimate partner, often brings the mixing of sexual partner due to nature of sex work, which in this paper is coined as "sexual mixing". Due to the desire to be protected in the male dominated society, many of these women practice unsafe behaviour with the regular partners to ensure their support, by making the behaviour a symbol of trust for their partners. In other cases, lack of authority or negotiation power to use protection with intimate partner and the fear of losing the intimate bond may scare a sex worker to engage in expected behaviours without an apprehension for her own health. All these social, emotional and psychological factors form a convoluted nexus, putting the safe sexual behaviour in these relationships at stake. Hence, it can easily be said that determining the factors involved in making decisions pertaining to sexual behaviour in intimate partner relationships is one of the greatest challenges for curbing STI and HIV today.

One of the most widely studied social cognition theories to understand this and many other psychological issues of public health importance is the Theory of Planned Behaviour (TPB) [8], in the context of adopting safe sexual behaviour. The historical antecedents of the TPB lie in the theory of reasoned action $[9,10]$. The theory identifies the proximal determinant of behaviour as the strength of an intention of engaging/not engaging in that behaviour. In turn, behavioural intentions are derived from two parallel cognitive processes. The first involves consideration of the individual's own attitudes towards the behaviour. The second involves consideration of the relevant behavioural norms. Personal attitudes are derived from measures of beliefs about the behaviour under consideration and values attached to those beliefs. Behavioural norms also comprise two elements: an appraisal of the likelihood that salient others would wish the individual to engage (or not) in the behaviour under consideration, and their motivation to comply with these expectations. Wee et al, 2004 used TPB to study the determinants of inconsistent condom use. They state that individuals are more likely to use condoms when they perceive the threat of AIDS to be serious and feel personally vulnerable to contracting it [11]. They should also have high self-efficacy in condom use and feel that it is an effective means of reducing the chance of HIV infection. In addition, attitudes towards condom use should be favourable and the perceived social norms for condom use supportive $[8,12]$. However, in situations where severity and vulnerability are high but self-efficacy and responseefficacy low, maladaptive behaviours may be adopted instead [13]. This theory has been effectively used to study and explain behaviours in various settings with varied populations and ethnic groups.

In the context of the discussion held in the above write-up, based on the existing pool of literature, Nepal exhibits an identical context for the risks and vulnerabilities for its female population. In the past few decades, the vulnerability for rapid transmission of HIV/AIDS in Nepal has increased manifold due to pervasive poverty coupled with low literacy, gender inequality, labour migration, female trafficking and increasing sex trade. Since Nepal's first case of HIV/AIDS was reported in 1988, unprotected sex has been one of the major drivers of the epidemic. Commercial sex work remains the primary route of STIs and heterosexual HIV/AIDS transmission-with multi-partner behaviour being the key factor for occupational vulnerability-and poses serious and detrimental repercussions on the overall sexual health of the populations at risk.

A cross-over between work and intimate relationship occurs among FSW and therefore, service providers need to look beyond the sex workers' working lives, and understand the influence of their profession and intimate relationships on their lives and overall health, including remaining disease free. Coupled with occupational vulnerability, the high risk behaviour in intimate relationships puts these women and their male partners at an elevated risk of acquiring infection through sexual transmission. It is against this backdrop, that this paper attempts to analyse the prevalence of STI arising from intimate partner relationships, among FSWs in Nepal.

\section{Specific Objectives of the Study}

- To understand the following behaviour patterns among FSWs having intimate partners in Nepal:

- concurrency and sexual mixing in FSWs 
- the major covariates of STIs in intimate partner sexual relationships among FSWs, and

- To study the STI treatment seeking behaviours of FSWs in Nepal

\section{Data and Methodology}

The data used in this paper was gathered from the behavioural survey conducted among selected FSWs, as part of the mapping and size estimation exercise of Most-At-Risk-Populations (MARPs), carried out in Nepal. A total of $2901 \mathrm{FSWs}$ were interviewed following a modified time-location sampling approach, out of which 1401 FSWs reported having intimate partner sexual relations in the last 12 months prior to the survey. For this paper, data of the 1401 FSWs with intimate partner sexual relations was used.

In the mapping and size estimation exercise, a scientific methodology, having comprehensive protocols was developed after various rounds of national consultations. This study was conducted in a participatory manner by involving people from the MARP community, thus making the process more inclusive, empowering and thereby enhancing the quality of data. Qualified local MARP community members were trained to function as field researchers. To enable maximum reach to the population, social mapping of key populations was done by adopting a "geographical approach" in which "population of target group", "risk activities" were defined clearly, and then locations where these activities take place were identified to capture hidden populations. To get a comprehensive picture of vulnerabilities and understand the core issues that are critical for these groups, data was collected at three stages, through primary key informants (those engaged directly in High Risk Activities (HRA), e.g., FSWs, MSMs and IDUs); secondary key informants (those closely associated with primary informants, e.g., pimps); and tertiary key informants (those who knew about MARPs and were usually involved with the secondary stakeholders, or working for (or against) the interests of the primary stakeholders e.g., international and national non-government organizations (INGOs and NGOs), government organizations, police). In the present context "Sexual Mixing" refers to the sexual act with multiple partners, particularly husband or long term intimate partner of FSWs is considered as long term partner and the clients, either regular or occasional are the other partners. Therefore, sexual involvement with both husband and clients is referred as "sexual mixing".

After broad mapping all hotspots of a location using the selfinstructional guidelines, field research teams collected qualitative information on different categories of MARPs present in the hot spots, the busiest days, their mobility patterns, average number of clients on daily and weekly basis, most accessed services, etc., for the last seven days as well as for the last 12 months. For this, field teams conducted group discussions with mixed categories of Key Informants (KIs), using the Participatory Rapid Appraisal (PRA) technique. To collect further information, in each hotspot, the research teams also interviewed a minimum of five MARPs, using a tool designed especially for the study.

In order to ensure data quality and further strengthen it, a four pronged strategies were adopted. These strategies were namely, selection of skilled research staff, uniform training to all the research teams, three tier monitoring and supervision, and revalidation of the mapping and size estimation exercise findings in a total of 10 percent of the selected locations.

\section{Results}

An examination of the background characteristics of FSWs who reported to have intimate partners showed that the majority (fourfifths) of these women were above the age of 20 years. A little more than two fifths belonged to the age group of 20-29 years, while the rest were aged 30 years or more This clearly shows that younger women (below age of 20 years) did not have any regular long term intimate partner. The reason may vary from changing norms in sex worker community or due to believe that greater support is required largely at later ages. The exact reason needs further exploration. Illiteracy was generally high in this population. A total of 55 percent of the FSWs having an intimate partner in the last 30 days reported to be illiterate (Table 1). A little over 20 percent or one-fifths of FSW had formal education up to 5 years and little less than 20 percent or one-fifth had 6-10 years of education. Higher secondary education was negligible in this group, may be because of the early school dropout due to financial crisis and poverty, or to take up sex work as a profession. Interestingly, 86 percent of the FSWs who reported to have an intimate partner in last 30 days also reported to be married, which implies that for a large majority of these women, the intimate partners were their husbands placing them at the higher risk of sexual mixing (Table 2). Also, about 80 percent, or four-fifths of these women reported to be migrants to the location. Although the fact that this may be to a great extent due to marriage migration is not ruled out, as most of these women are married, but it indicates that these women entered sex work profession after they got married, and at their marital town.

\begin{tabular}{|c|c|c|}
\hline \multicolumn{2}{|c|}{ Background Characteristics } & \multirow{2}{*}{$\begin{array}{c}\text { Percent } \\
17.1\end{array}$} \\
\hline Age & Below 20 years & \\
\hline & 20-29 years & 43.8 \\
\hline & 30 years and above & 39.1 \\
\hline \multirow[t]{4}{*}{ Education } & Illiterate & 55.4 \\
\hline & Up to 5 years & 21.3 \\
\hline & $6-10$ years & 18.6 \\
\hline & 10 years and above & 4.7 \\
\hline \multirow[t]{2}{*}{ Marital status } & Married & 86.5 \\
\hline & Unmarried & 13.5 \\
\hline \multirow[t]{2}{*}{ Migration status } & Migrant & 80.1 \\
\hline & Non-migrant & 19.9 \\
\hline
\end{tabular}

Table 1: Profile of FSWs having intimate partner relationship in the last 30 days prior to the survey by some selected background characteristics, Nepal, 2010-11.

\begin{tabular}{|c|c|c|c|c|}
\hline \multicolumn{2}{|c|}{ Background Characteristics } & \multicolumn{3}{|c|}{ Coital Frequency in the last 30 Days } \\
\hline & & less than 7 & 7 to 15 & 15 or more \\
\hline \multirow[t]{3}{*}{ Age } & Below 20 & 26.9 & 28.6 & 44.5 \\
\hline & $20-29$ & 17.9 & 28.9 & 53.3 \\
\hline & 30 and above & 17.9 & 32.2 & 49.9 \\
\hline \multirow[t]{4}{*}{ Education } & Illiterate & 16.5 & 29.2 & 54.3 \\
\hline & up to 5 years & 24.1 & 32.7 & 43.2 \\
\hline & 6 to 10 years & 23.1 & 28.5 & 48.5 \\
\hline & 10 and above & 18.2 & 36.4 & 45.5 \\
\hline \multirow[t]{2}{*}{ Marital status } & Married & 17.8 & 30.5 & 51.7 \\
\hline & Unmarried & 29.7 & 28.1 & 42.2 \\
\hline \multirow[t]{2}{*}{ Migration status } & Migrant & 19.6 & 30.9 & 49.5 \\
\hline & Non-migrant & 18.8 & 27.1 & 54.1 \\
\hline \multirow{2}{*}{$\begin{array}{l}\text { Age at entry into } \\
\text { sex work }\end{array}$} & below 16 years & 19.7 & 26.3 & 54.1 \\
\hline & 16 years or above & 26.8 & 30.0 & 43.3 \\
\hline Total & & 19.4 & 30.1 & 50.4 \\
\hline
\end{tabular}

Table 2: Percent distribution of FSW having intimate partner sexual relations by their coital frequency in the last 30 days prior to the survey and some selected background characteristics, Nepal, 2010-11. 
Sexual risk is an occupational hazard of this profession. Sexual risk taking and high risk behaviour is in some cases a necessity, and in others an obligation for those in this profession. Multi-partner, unprotected sex etc., are the bi-products of this trade which serve as the determinants or intermediate factors for sexual health, along with infections including STIs and HIV/AIDS. In this framework of transmission, "sexual mixing" is also an important component to explain the STI transmission dynamics. "Sexual mixing" operates as a social bridge and studying it provides insights into the types of persons that come into contact, within and across risk groups. A study of "sexual mixing" in this population revealed that about 40 percent or two-fifths of FSWs in Nepal had commercial as well as non-commercial sexual partners. Further, it was seen that partner mixing was considerable even in non-commercial sexual partners. Results revealed that 5.2 percent of the FSWs reported having more than one non-commercial partner along with commercial partners.

Coital frequency is also recorded as an important intermediate factor for STI and HIV. Literature shows that higher coitus interactions can be detrimental to women's health. The condition of FSWs with respect to this factor is worse, as they are not just exposed to high coital frequency but also multi-partner and many times unprotected coitus. Even an empowered and mobilized FSW, who is equipped with all the possible protective measures, including condoms and a strong negotiation power to use it with commercial partners, may engage in regular unprotected sex with an intimate partner. The intimacy and fear of losing the partner may compel these women to do so, increasing their vulnerability to STI/HIV manifold. An attempt was made to study the coital frequency of FSWs with intimate partners, where coital frequency was measured in terms of number of sexual encounters in last 30 days before the survey. Data was analysed under three categories namely, less than 7 times, 7 to 15 times, and 15 or more times. The results showed that over half the FSWs having intimate partner relationships in the last 30 days prior to the survey reported to have a frequency of 15 or more coituses in the last 30 days. Another 30 percent reported to have 7 to 15 and about 20 percent or one-fifths of women reported less than 7 coituses in last 30 days. Age, education, Marital status and age at entry into sex work are significantly $(\mathrm{P}<0.01$ and $\mathrm{P}<0.05)$ associated with coital frequency in last 30 days. Those who were illiterate married, operating from usual place of residence, and had entered in this profession before 16 years of age were more likely to have a higher coital frequency than their counterparts. Interestingly, It was further observed that coital frequency with intimate partner was low among women below 20 years of age ( 27 percent), unmarried (30 percent) and those who had entered the profession after completing 16 years of age.

Marital status and coital frequency seems to have a significant effect on the condom use behaviour of the FSWs. It was seen that unmarried FSWs were 0.3 times $(\mathrm{p}<0.01)$ less likely to use condoms than their counterparts. At the same time, for those who had a coital frequency of 7 to 15 coituses in the last 30 days, and those who have more than 15 coituses in a week, it was $0.6(\mathrm{p}<0.05)$ and $0.5(\mathrm{p}<0.01)$ respectively. As discussed earlier, protected sex with intimate partner is affected by various emotional, psychological, social, and economic factors. The combination of unprotected sex and multi-partner relations by even one of the intimate partner may result in transmission of infection from self to the partner, and from one to other partners creating a convoluted web of transmission. Use of condoms can go a long way as a singlehanded strategy in cutting this transmission. To assess the current scenario of condom use in intimate partner relationships, the condom use in the last sex was recorded. Age of the FSWs $(\mathrm{P}<0.01)$, Education
$(\mathrm{P}<0.05)$, marital status of the FSWs $(\mathrm{P}<0.00)$ and age at entry into sex work $(\mathrm{P}<0.05)$ are significantly associated with condom use in last sex. Over half of the FSW respondents reported not using condom in the last sex with their intimate sexual partners the proportion of such FSWs was significantly higher among illiterates, those aged 30 years and above, currently married, and having relatively lesser coital frequency with the intimate partner. At the same time, it is also important to highlight here that condom use was higher among younger FSWs. The reason for this finding may be that the relationship with the intimate partner is not of a marital bond. Supporting this argument is another finding that 69 percent of unmarried FSW are using condoms with their intimate partners. This underlines the fact that with for FSW who are married, the condom is a treated as a means of contraception and not a protection against STIs. Education also showed a positive contribution in condom use as about 60 percent or three-fifths of FSWs educated for 10 years or more reported to be using condoms.

The sexually transmitted infections are quite common in sex workers but literature proves that safe sexual practices can significantly reduce their prevalence, even in high-risk population of FSWs. STIs have a high prevalence in FSWs in Nepal. Over one-third of FSWs reported to suffer from STIs in the last 12 months. Age $(\mathrm{P}<0.01)$, education $(\mathrm{P}<0.01)$ and marital status $(\mathrm{P}<0.01)$ seem to have significant effect on the STI prevalence among this group (Table 3 ). The proportion of STI cases in FSWs aged 30 years or above ( 44 percent) was almost twice the amount in FSWs aged less than 20 years (25 percent). Also, STI prevalence was found to increase with increasing age, which could be attributed to higher prevalence of condom use or lower coital frequency among younger FSW compared to older FSWs, as noted earlier, which were protecting them against STI. Further, the level of education showed a very profound effect on STI prevalence. The prevalence among illiterate was found to be four times the prevalence among those having more than 10 years of education. It is not surprising to see that the educated group demonstrated better performance in terms of all the indicators of safer behaviour. Further, the gap in health conditions of literates and illiterates in terms of STI prevalence was overwhelming. It is also

\begin{tabular}{|c|c|c|c|}
\hline \multicolumn{2}{|c|}{ Background Characteristics } & \multicolumn{2}{|c|}{ Condom use in last sex } \\
\hline & & Yes & No \\
\hline \multirow[t]{3}{*}{ Age } & Below 20 years & 58.0 & 42.0 \\
\hline & 20-29 years & 48.1 & 52.0 \\
\hline & $\begin{array}{l}30 \text { years and } \\
\text { above }\end{array}$ & 45.7 & 54.3 \\
\hline \multirow[t]{4}{*}{ Education } & Illiterate & 45.9 & 54.1 \\
\hline & up to 5 years & 52.2 & 47.9 \\
\hline & 6 to 10 years & 50.8 & 49.2 \\
\hline & 10 and above & 60.6 & 39.4 \\
\hline \multirow[t]{2}{*}{ Marital status } & Married & 45.7 & 54.3 \\
\hline & Unmarried & 68.8 & 31.3 \\
\hline \multirow[t]{2}{*}{ Migration status } & Migrant & 47.9 & 52.1 \\
\hline & Non-migrant & 52.4 & 47.6 \\
\hline \multirow{2}{*}{$\begin{array}{l}\text { Age at entry into } \\
\text { sex work }\end{array}$} & Below 16 years & 46.8 & 53.2 \\
\hline & 16 years or above & 47.2 & 52.8 \\
\hline \multirow{3}{*}{$\begin{array}{l}\text { Coital frequency in } \\
\text { the last } 30 \text { days }\end{array}$} & Less than 7 & 46.2 & 53.9 \\
\hline & 7 to 15 & 47.0 & 53.0 \\
\hline & More than 15 & 51.0 & 49.0 \\
\hline Total & & 47.1 & 52.9 \\
\hline
\end{tabular}

Table 3: Percent distribution of FSW having intimate partner sexual relations in the last 30 days prior to the survey by Safe sexual practices and some selected background characteristics, Nepal, 2010-11. 
important to highlight here that STIs were significantly higher among married FSWs as against the unmarried ones. Literacy and migratory status were found to significantly affect the prevalence of STIs (Table 4). It was seen that non-migrants were 0.7 times $(\mathrm{p}<0.05)$ less likely to have STI as against migrants. Logistic regression analysis also highlighted a negative impact of years of education on STI prevalence, as FSWs having up to 5 years of education been found to be 1.4 times more likely to have an STI. Similarly, those with up to 10 years and those with more than 10 years of education were found to be 2.3 times and 4.5 times respectively more likely to have STI as compared to those with no formal education. Although, these findings seem to contradict other documented literature, it could be attributed to the fact of under reporting of STIs by illiterate FSWs due to lack of awareness, leading to them being unable to recognize STI symptoms, and/or not seeking medical aid.

An enquiry into the treatment seeking behaviour of FSW respondents showed an overall high prevalence of treatment seeking for any STI related symptom in the last 12 months (Table 5). Overall, 92 percent of the respondents reported to have sought treatment for their STIs or related symptoms. Although some variation was observed among some sections of the group, the level of treatment remained near 90 percent except for those women who reported to have early entry into sex work. It was seen that only four-fifths or about 80 percent of FSWs who entered the profession before the age of 16 years sought any medical treatment for STIs. Underlying factors for this can be less empowerment of these women or lack of knowledge about self-health care, etc. Although analysis of the reasons behind this finding is beyond the scope of this paper, it definitely highlights a need to study the entry of minor girls into sex trade and also the challenges that early initiation to sex work poses to the later life of FSWs. In order to optimally utilize any service, accessibility, affordability and availability play a very critical role. They are the direct measures of the quality, supply and demand of service. Similarly, for a population like FSW, it is very important to know the level of service utilization and the preferred service delivery points.

\begin{tabular}{|l|l|l|l|}
\hline \multicolumn{2}{|c|}{ Background Characteristics } & \multicolumn{2}{c|}{$\begin{array}{c}\text { Ant STI in last 12 } \\
\text { months }\end{array}$} \\
\hline & & Yes & No \\
\hline Age & Below 20 years & 24.5 & 75.5 \\
\hline & $20-29$ years & 31.8 & 68.2 \\
\hline Education & 30 years and above & 43.5 & 56.5 \\
\hline & Illiterate & 42.7 & 57.3 \\
\hline & Up to 5 years & 33.3 & 66.7 \\
\hline \multirow{2}{*}{ Marital status } & 6 to 10 years & 20.0 & 80.0 \\
\hline & 10 and above & 12.1 & 87.9 \\
\hline Migration status & Married & 37.1 & 63.0 \\
\hline \multirow{2}{*}{ Age at entry into sex work } & Unmarried & 22.4 & 77.6 \\
\hline & Migrant & 34.4 & 65.6 \\
\hline Coital frequency & Non-migrant & 37.3 & 62.7 \\
\hline & 16 years 16 years above & 35.3 & 64.7 \\
\hline & Less than 7 & 35.3 & 64.7 \\
\hline Condom use in last sex & 7 to 15 & 34.4 & 65.6 \\
\hline & More than 15 & 35.8 & 65.3 \\
\hline Total & Yes & 34.7 & 64.6 \\
\hline & No & 35.4 & 65.3 \\
\hline
\end{tabular}

Table 4: Percent distribution of FSW having intimate partner sexual relations in the last 30 days prior to the survey by prevalence of STI and some selected background characteristics, Nepal, 2010-11.

\begin{tabular}{|c|c|c|c|}
\hline \multicolumn{2}{|c|}{ Background Characteristics } & \multirow{2}{*}{$\begin{array}{c}\text { Condom use } \\
\text { Exp ( } \beta)\end{array}$} & \multirow{2}{*}{$\begin{array}{l}\text { Any STI } \\
\text { Exp ( } \beta \text { ) }\end{array}$} \\
\hline \multirow{4}{*}{ Age } & & & \\
\hline & Below 20 years ${ }^{\circledast}$ & & \\
\hline & 20-29 years & 1.045 & 0.967 \\
\hline & 30 years and above & 1.036 & 0.743 \\
\hline \multirow[t]{4}{*}{ Education } & Illiterate ${ }^{\circledR}$ & & \\
\hline & Up to 5 years & 0.854 & $1.445^{\star *}$ \\
\hline & 6 to 10 years & 1.086 & $2.364^{* * *}$ \\
\hline & 10 years and above & 0.84 & $4.596^{* * *}$ \\
\hline \multirow[t]{2}{*}{ Marital status } & Married $^{\circledR}$ & & \\
\hline & Unmarried & $0.375^{\star \star \star}$ & 1.222 \\
\hline \multirow[t]{2}{*}{ Migration status } & Migrant $^{\circledR}$ & & \\
\hline & Non-migrant & 0.915 & $0.794^{* *}$ \\
\hline \multirow[t]{3}{*}{ Coital frequency } & Less than $7^{\circledR}$ & & \\
\hline & 7 to 15 & $0.696^{* *}$ & 1.048 \\
\hline & More than 15 & $0.589^{* * *}$ & 1.049 \\
\hline \multirow[t]{2}{*}{ Age at entry into sex work } & Below 16 years & & \\
\hline & 16 years or above & 0.978 & 0.89 \\
\hline \multirow[t]{2}{*}{$\begin{array}{l}\text { Condom use in with intimate } \\
\text { partner }\end{array}$} & Yes $^{\circledR}$ & & \\
\hline & No & & 1.092 \\
\hline
\end{tabular}

Table 5: Logistic regression odds ratios showing adjusted effects of sociodemographic and behavioural factors on condom use in the last sex and prevalence of STIs among FSWs in Nepal, 2010-11.

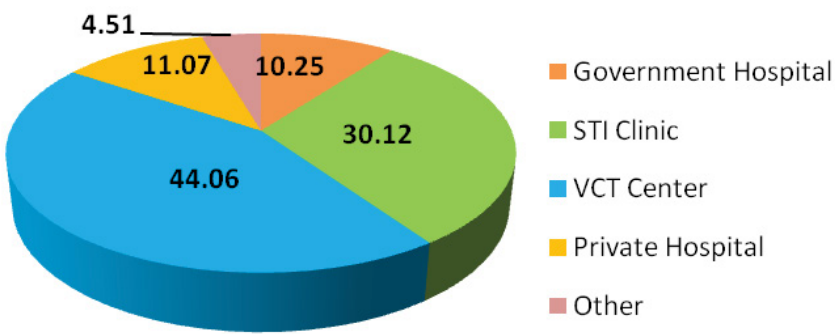

Figure 1: The most preferred STI treatment source by FSWs having intimate partner relationship.

Secondly, to achieve high levels of health care seeking, treatment, and follow-up, it is important to know and further strengthen the health care delivery systems in the areas. Among this group of women, the most preferred STI treatment sources were VCT centres, followed by STI clinics, private hospitals and government hospitals (Figure 1).

\section{Discussion}

Sex work in Nepal is illegal still there are approximately 25,00034,000 active commercial sex workers in the country (2009). The data from the BSS survey in Nepal shows that 30 percent of all female sex workers are below the age of 18 years. Although sex trade is under judicial restrictions, data reveals that sex work is driven by demand with an extremely high client-FSW ratio of 20:1 [14]. In Nepal, FSWs are considered reservoirs of STIs. Not only they, but their partners also face certain vulnerabilities. The partners of FSWs are at risk of becoming infected with STIs themselves, and/or act as bridge to other sex partners who could also be from the general population [14].

The relationship dynamics of FSW and intimate partners are very complex and eventually ended into mixing sexual partners which is cited as "Sexual Mixing". Although sex work is a demand driven profession with a very high client load, the need for an intimate bond and an intimate partner relationship among FSWs is also high, as shown by 
the present study. A thorough understanding of the various dynamics of intimate partner relationship in a FSWs life is needed to study the sexual behaviour of FSWs and their partners in these relationships. An intimate partner provides emotional support, social security, feelings of inclusion, a shield protecting FSWs from societal harassments, family, secrecy (especially for home based FSW), procreation, etc. The study shows high prevalence of unsafe sexual behaviour practiced by both FSWs and their partners, whether married or unmarried regular partners in intimate relationships. Although the prevalence is much higher among married couples, but the unmarried bonds cannot be ruled out as partner mixing in intimate partners is higher in the relationships out of wedlock. Some of the factors that may be leading to the unsafe behaviour with the regular partner are "trust" to maintain the support of the partner by practicing the expected behaviour, "power dynamics" which result in lack of authority or negotiation power with the FSW to use protection with intimate partner and, "romantic attachment anxiety" or fear of losing the intimate bond. In this section we attempt to use the theory of planned behaviour to explain behaviour and identify factors associated with sexual behaviour and condom use.

The theory of planned behaviour studies various facets and predicators of actions to understand the final behavioural outcome. In our study we could map how the behavioural intentions were significantly predictable from attitudes, subjective norms and perceived behavioural control. An individual is more likely to behave in a certain way towards a certain therapy when they perceive the threat to be serious and feel personally vulnerable to contracting it. In our study, the prevalence of regular condom use in FSWs aged 30 years or above was less than half compared to in younger FSWs. Interestingly, the majority of older women had their intimate partners as their husbands. In this relationship the perception of risk as well as the behaviour control is low. The subjective norms, i.e. the expectations of behaviour from the partners towards themselves, also play a very critical role. Interaction among these predictors further shapes the intensions and results into certain behaviour, which in this case is absence of condom use with the intimate partner. Another important aspect of theory of planned behaviour is perceived self-efficacy. FSWs should also have high selfefficacy in condom use, and feel that it is an effective means of reducing the probability of acquiring HIV infection. In case of FSWs included in the study, the findings clearly pointed towards the fact that condom use was treated merely as a means of contraception, and not of disease prevention. These findings defeat the notion of condom use induced by self-perceived efficacy. The theory of planned behaviour also highlights the need for favourable attitudes towards safe sexual behaviour and supportive perceived social norms for condom use. In case of FSWs in Nepal, the perceived social norms for condom use were supportive in case of commercial partner. However, with an intimate partner the women's own perceptions about safe sexual behaviour and were not found to be shaping her intentions and behaviour, but rather the subjective norms of partner expectations, that is, her beliefs about what her partner thought she should do shaped her behaviour. For example, the regular partner's desire to procreate was found to have an upper hand on the normative beliefs of an FSW. This was further worsened by the FSWs need for intimacy and security, leading to a situation where severity and vulnerability were high but self-efficacy and response efficacy were low, thus, leading to adoption of maladaptive behaviours instead.

\section{Conclusion and Recommendation}

There is an unambiguous void of information available on this population of Nepal. The literature review corroborates this lack of documented evidences that can speak for this population, leaving many issues unsolved, many unanswered, and rest only debated. The considerable population size of this community, increasing HIV prevalence in Nepal, and deteriorating health conditions of these highly vulnerable and socially disadvantaged women demands serious and urgent attention of both researchers and programme managers. This section is an attempt to identify and flag some relevant issues of these women along with contributing to increase the evidence base for effective and efficient programme planning.

The study underscores the need to analyse the sexual networks and the pattern of HIV/STIs that exist in the FSW community prior to the introduction of interventions, in order to understand exactly who to target. Second, it also shows that the coverage of interventions needs consideration for an appreciable impact on STI prevalence in the community. There may not be a noticeable effect within the general population if enough individuals and their partners do not participate in the programme.

The FSW community of Nepal may be witnessing some changing norms in terms of relative empowerment and access to information for more women, but at the same time, distribution of change in this community has been observed to be unequal, where the younger group is performing better than their older counterparts. Most of the younger FSWs did not report being married or having an intimate partner, and also reported lower coital frequency along with higher condom use and safer sexual behaviour, but situations remain challenging for the FSWs aged more than 20 years. Most of these women reported having an intimate partner who may be primarily for support required at later ages. Although a large majority of these partners are husbands, but the risky behaviour and magnitude of vulnerability only multiplies for these sex workers. Among the underlying factors operating for the vulnerability of this population may be many social, economic or familial factors like migration, poverty, unemployment, etc. Illiteracy was observed to be very high in this population, generating a considerable proportion of unskilled labour force. High rates of early school dropouts due to financial crisis and poverty or early marriage can be factors playing a significant role here. Nevertheless, literacy is showing profound positive impact on safe sexual behaviour of these women including on use of condoms and STI prevalence.

Considering the above laid arguments, a sound strategy to tackle the vulnerabilities of these women can be their education. The impact of education is being observed in the form of the safe sexual behaviour by empowering these women, making them more aware, and probably also improving their negotiation skills. The women of higher ages need special attention to reduce their risk exposure. Counselling of these women and behavioural change communication (BCC) with them has been a proven programme strategy. As most of these women are cohabitating with husbands, it may be possible that their professional status is hidden. In such cases, to ensure their optimum utilization, programmes or services should be designed considering the sensitive issue of confidentiality of home based sex workers. As home-based sex work usually works in form of hidden network, identifying these networks, planning and placing the services for them becomes a challenge for programme managers. Therefore, peer based models can be useful in enhancing behaviour change and ensuring safe sexual practices. Along with services, working knowledge, especially on sexual and reproductive health, safe sexual practices, condom use, etc., should be given to FSWs. Self-help or community based support groups for women should be created to generate additional economic opportunities. 
The entire condom campaign and BCC is heavily influenced by interpersonal relationship between the sexual partners. In a conservative society like Nepal, where women are subjugated while males hold an upper hand on decisions pertaining to sex and procreation condoms are merely seen as contraceptives and not a protection against STIs. In Nepal, the vast majority of married FSWs do not use condoms, resulting in high prevalence of STIs in this community, especially among older and married FSWs. The relatively wider use of condoms among younger FSWs is protecting them against STIs and allowing them to lead healthy lives. The higher incidence of STIs among married FSWs highlights the need to work on intimate partner relationships and empower women in order to improve their condom negotiating power, and enable them to bargain on condom use with their husband or regular partner. It also highlights the need for innovative campaigns for condom use and for addressing any stigma related to condom use intimate relations. There is a need to project condom as a means of pleasure stimuli and not just of family planning. This fact again highlights the need to work on intimate partner relationship and empower women to improve their condom negotiating power which will enable them to bargain condom use with their husband or regular partner.

To achieve long term sustainability of any programme or service, it is important to make it an integral part of public health system. The findings of the study and the discussion also highlights that the onus of providing better services and health to FSWs lies on the country's public health system. Specific strategies to achieve the programme and service objectives for this group may be developed keeping in mind the following needs:

\section{- Intensified free condom distribution}

- Massive awareness and BCC interventions to ingrain safe practices in sexual behaviour

- Peer educators, outreach services and mobile clinics

- Expansion of functional ICTCs

- Institutionalization of the Rights Based Approach to health care delivery system.

\section{References}

1. UNAIDS, UNDP, UNFPA, ICAAPIX (2009) HIV and Intimate Partner Transmission: A Call for Action. 9th International Congress on AIDS in Asia and the Pacific Symposium Report.

2. Commission on AIDS in Asia (2008) Redefining AIDS in Asia: Crafting an Effective Response. Report of the Commission on AIDS in Asia. Oxford University Press.

3. Caitlin Wiesen (2009) UNDP Regional Centre for Asia and the Pacific (from China HIVIAIDS Survey Data, UNDP.

4. Silverman JG, Decker MR, Saggurti N, Balaiah D, Raj A (2008) Intimate partner violence and HIV infection among married Indian women. JAMA 300: 703-710.

5. Prasada Rao, UNAIDS Regional Support Team for Asia and the Pacific quoting Decker (2009) Intimate partner violence is both a risk marker and a transmission mechanism for women's HIV infection: Findings from a national population-based sample of Indian husband-wife dyads. J AIDS.

6. Geeta Rao (2000) HIV and Intimate Partner Transmission: A Call for Action Symposium Report.

7. NafisSadik (2009) UN Secretary General's special envoy on HIV/AIDS in Asia and the Pacific. 9 $^{\text {TH }}$ International Conference on AIDS In Asia (Icaap) Bali, Indonesia.

8. Ajzen I (2011) The theory of planned behaviour: reactions and reflections. Psychol Health 26: 1113-1127.

9. Bledsoe LK (2006) Smoking cessation: an application of theory of planned behavior to understanding progress through stages of change. Addict Behav 31: 1271-1276.

10. Schroeder SA, Fulton DC (2013) Public lakes, private lakeshore: modeling protection of native aquatic plants. Environ Manage 52: 99-112.

11. Wee S, Barrett ME, Lian WM, Jayabaskar T, Chan KW (2004) Determinants of inconsistent condom use with female sex workers among men attending the STD clinic in Singapore. Sex Transm Infect 80: 310-314.

12. Sheeran P, Taylor S (1999) Predicting intentions to use condoms: a metaanalysis and comparison of the theories of reasoned action and planned behaviour. J ApplSocPsychol 29: 1624-1675.

13. Rogers RW, Prentice-Dunn S (1997) Protection motivation theory. In: Gochman DS, (Ed.), Handbook of health behavior research. 1: Personal and social determinants. Plenum Press, New York 113-132.

14. NCASC (2011) Integrated Biological and Behavioural Surveillance Survey among Female Sex Workers Kathmandu Valley, 2008, Round III. 